\title{
喷墨打印高精度图案研究进展
}

\author{
耶旻噮王京霞＼cjkstart王利涁＼cjkstart宋延林* \\ (中国科学院化学研究所 北京 100190)
}

\begin{abstract}
摘要 近年来, 功能材料的图案化及其在高性能光电器件的应用研究受到了广泛关注. 与传统图案化方法相比, 喷墨 打印技术更容易实现大面积复杂图案的直接书写和复合功能材料的图案化, 且制备简便, 成本低廉, 使其成为最受关 注的图案化方法之一. 综述了近年来喷墨打印制备高精度图案的研究进展. 包括通过优化墨滴的化学组成、调控基材 的化学或物理结构以及改进喷墨设备等方法以提高喷墨打印分辨率; 以及通过控制液滴内部的毛细流动和三相接触 线的移动抑制喷墨液滴的 “咖啡环” 效应, 以实现均质打印. 文章最后展望了喷墨打印制备高精度图案的研究发展方 向. 这些工作对于实现高性能器件的制备具有重要意义.
\end{abstract}

关键词 喷墨打印; 图案化; 高精度; 咖啡环

\section{Research Progress of High-precision Patterns by Directly Inkjet Printing} Kuang, Minxuan Wang, Jingxia Wang, Libin Song, Yanlin*

(Laboratory of New Materials, Institute of Chemistry, Chinese Academy of Sciences, Beijing 100190)

\begin{abstract}
High-precision patterns have aroused wide attention due to their important applications in high-performance optical/electrical devices. By virtue of easy and direct writing of desired patterns, high throughput and low-cost, inkjet printing has become one of the most promising candidates for the manufacture of patterns, compared with conventional approaches such as photolithography, microcontact printing. Various materials can be precisely deposited on target positions. As a primary focus, improving the precision of printed patterns would greatly enhance the performance of the devices. So far, various approaches have been developed for improving the printing precision. Pattern resolution is limited by the volume of jetted droplet and the area of droplet spreading on substrate. Finer resolution can be achieved through reducing the deposition dimension, i.e. optimization of the chemical composition of ink, chemical/physical modification of substrates, and improvement of printing apparatus. Besides, uniform deposition of functional materials is also essential for fabricating high-precision patterns and high-performance devices. "Coffee ring" is a common phenomenon during the drying process of droplets, which is generated due to the pinning three phase contact line (TCL) and the outward capillary flow in an evaporating droplet. Therefore, homogenous deposition without "coffee ring" effect can be realized by tuning the capillary flow in the droplet and controlling the movement of the TCL as the droplet evaporating. In this paper, we presented the recent research progress for achieving high-precision patterns by inkjet printing, including minimizing the deposition dimension to improve the resolution and avoiding the "coffee ring" effect to achieve high uniformity. These reports will probably promote the development of novel and facile method for high-precision inkjet printing. New trends and research perspectives were briefly discussed at the last section of this review.
\end{abstract}

Keywords inkjet printing; patterning; high-precision; coffee ring

\section{1 引言}

近年来, 高精度图案化在高性能微半导体、电子器 件以及生物功能材料等的制备方面的研究受到了广泛 关注 ${ }^{[1 \sim 3]}$. 实现图案化的方法有光刻蚀法 ${ }^{[4]}$ 、电子束刻蚀 法 $^{[5,6] \text { 、微接触转印法 }}{ }^{[7,8]}$ 以及喷墨打印法 ${ }^{[9,10]}$ 等. 传统的 光刻蚀法、电子束刻蚀法以及微接触转印法的制备工艺 复杂且成本较高, 使其在大面积图案化方面的应用受到 限制. 而喷墨打印技术更容易实现大面积复杂图案的直
接书写和复合功能材料的图案化，且制备简便，成本低 廉，使其成为最有前景的图案化方法之一. 目前，喷墨 打印技术已经被广泛应用于各类功能器件的制备. 高精 度图案的喷墨打印技术可以实现墨滴的准确定位，对于 提高所得微图案的分辨率、改进微器件的功能至关重要. 然而, 由于喷孔直径的限制，使用现有普通喷墨打印机 所打印图案的最小点直径或最窄线宽只 $20 \sim 30 \mu \mathrm{m}$. 同 时，由于墨滴蒸发过程中的 “咖啡环” 效应，功能溶质 在墨滴的中心和边缘的沉积密度不同，降低了所制备图

\footnotetext{
* E-mail: ylsong@iccas.ac.cn

Received May 14, 2012; published August 17, 2012.
}

Project supported by the National Natural Science Foundation of China (Nos. 50973117, 21074139, 91127029, 51173190, 20904061, 50625312, U0634004, and 20721061) and 973 Program (Nos. 2009CB930404, 2011CB932303, and 2011CB808400).

项目受国家自然科学基金(50973117，21074139，91127029，51173190，20904061，50625312，U0634004，20721061)和 973 计划(Nos. 2009CB930404, 2011CB932303, 2011CB808400)资助. 
案的均匀程度. 这些缺陷很大程度上限制了喷墨打印技 术在高性能微器件制备方面的应用. 本文综述了近年来 喷墨打印制备高精度图案的研究进展, 包括提高喷墨打 印分辨率及抑制 “咖啡环” 效应.

\section{2 提高喷墨打印图案分辨率}

喷墨打印图案的分辨率通常由墨滴沉积面积决定. 通过优化墨水的化学组成、调控基材表面的化学组成或 物理结构以及改进喷墨设备等方法可以减少喷射墨滴 的尺寸或者控制墨滴在基材表面的浸润行为, 有效提高 喷墨打印图案的分辨率.

\section{1 优化墨水的化学组成}

墨水的化学组成直接影响喷射墨滴在基材表面的 浸润铺展行为, 进而影响喷墨打印图案的分辨率. 通常, 通过提高墨水的固含量或者加入高粘度溶剂增大墨水 粘度, 可以减小墨滴在基材表面的铺展面积. 然而若墨 水粘度过大，墨滴在喷墨打印过程中难以成形，同时墨 水通过喷孔时所受的阻力较大, 墨水无法从喷孔中喷 出. 为了克服这一矛盾, Schubert 等 ${ }^{[11]}$ 采用了一种热敏 性凝胶聚合物包覆二氧化钛粒子的墨水, 控制墨滴在基 材表面的铺展行为, 得到较窄的均匀连续线. 该墨水在 临界温度以下时, 聚合物链在溶剂中呈伸展状态, 墨水 粘度较低; 而在临界温度以上时, 聚合物链卷曲, 墨水 粘度迅速增大. 当粘度较低的墨滴喷射至加热的基材上 后, 其粘度迅速增大，从而抑制了墨滴在基材上的铺展 行为. 随后, 该课题组采用加热基材的方法控制墨滴在 基材表面的铺展行为, 在未处理的聚合物基材表面得到 了宽度为 $40 \mu \mathrm{m}$ 的连续均匀的导电线 ${ }^{[12]}$.

为实现喷墨打印制备亚微米尺度的高性能场效应 晶体管的应用要求, Sirringhaus 课题组 ${ }^{[13,14]}$ 通过调整墨 水的组成, 将修饰后的液滴作为基底, 提出了一种非刻 蚀、自校准的喷墨打印技术, 制备了宽度小于 $100 \mathrm{~nm}$ 的 通道. 如图 1a 所示, 由于第一滴液滴的低表面能作用, 第二滴液滴被排斥并自动流向侧面, 形成纳米尺寸的通 道. 他们采用了两种方法制备低表面能液滴, 一是在墨 水中添加了含氟表面活性剂, 液滴干燥过程中含氟表面 活性剂迁移至液滴表面组装, 降低了液滴表面能; 二是 将液滴用 $\mathrm{CF}_{4}$ 进行等离子体疏水化处理, 使之具有低表 面能.

\section{2 调控基材表面的化学组成或物理结构}

墨滴喷射至基材表面时往往会发生浸润铺展, 使墨 滴的沉积面积增大, 降低所制备图案分辨率. 通过优化 基材表面的化学组成或物理结构, 可以限制喷墨液滴的 铺展, 或者控制液滴的铺展方向, 实现高分辨率打印.

中科院化学所宋延林研究员课题组通过控制基材 的表面能, 喷墨打印制备了高质量的图案化光子晶体.

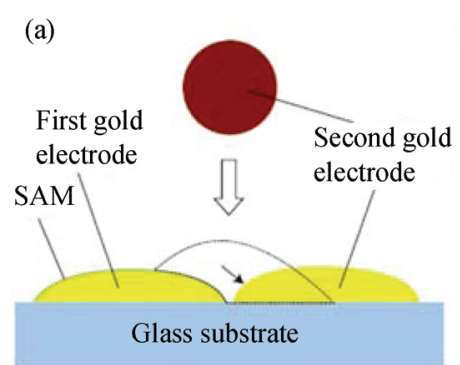

$2^{\text {nd }}$ gold $\quad 1^{\text {st }}$ gold

(b)

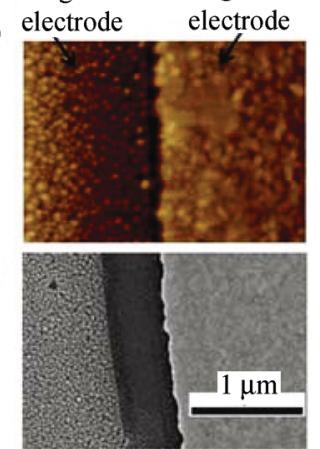

图 1 自校准喷墨打印. (a)过程示意图. 其中的虚线表示在疏水性的 第一电极表面, 第二滴金纳米粒子墨水去浸润前的位置. (b)和(c)分别 为所制备的第一电极与第二电极之间的窄通道的原子力显微镜照片 $(2 \mu \mathrm{m} \times 2 \mu \mathrm{m})$ 和扫描电子显微镜照片. 其中(b)图中的两条电极间通道 宽度为 $50 \mathrm{~nm},(\mathrm{c})$ 图中两条电极间通道宽度为 $400 \mathrm{~nm}$

Figure 1 Self-aligned inkjet printing (SAP). (a) Schematic of the SAP process. The dashed line indicates the initial position of the printed gold nanoparticle ink before dewetting from the hydrophobic surface layer on the first electrode. Atomic force microscopy $(2 \mu \mathrm{m} \times 2 \mu \mathrm{m})(\mathrm{b})$ and scanning electron microscopy (c) image of the short channel between a printed second and an evaporated first gold electrode. In (b) the channel length $L$ between the two electrodes is on the order of $50 \mathrm{~nm}$. In (c) print conditions were selected to produce a wider channel $L=400 \mathrm{~nm}$

他们考察了基材表面能对墨滴铺展行为以及光子晶体 组装行为的影响. 通过优化与墨水相匹配的基材, 得到 了分辨率和光学性能良好的图案化光子晶体 ${ }^{[15]}$. 为了 进一步减小墨滴在基材表面的铺展面积, 他们研究了多 孔氧化铝结构对液滴扩散的影响 ${ }^{[16]}$. 他们制备了单封 闭孔和通孔两种纳米孔的氧化铝膜, 系统研究了孔径对 液滴浸润性的影响，发现通过控制孔径可以使液滴渗透 至纳米孔中，从而有效限制墨滴的扩散行为. 在此基础 上，他们在具有微纳结构的亲水多孔氧化铝基材上打印 制备了高分辨的超亲油图案，实现了直接打印制 版 ${ }^{[17,18]}$, 克服了目前基于感光成像原理制版需要曝光、 冲洗带来的繁琐工艺和环境污染.

基材表面的物理化学结构不仅可以诱导墨滴向下 渗透，还可以控制墨滴在基材表面进行方向可控的浸 润. 剑桥大学的 Sirringhaus 课题组在亲水玻璃基底上构 建了聚酰亚胺的疏水图案，并利用疏水图案对墨滴的排 斥作用，打印制备了宽度为 $5 \mu \mathrm{m}$ 的通道 ${ }^{[19]}$. 随后该课 题组进一步利用刻蚀法在亲水基材上获得纳米尺寸的 疏水图案, 制备了最窄宽度为 $500 \mathrm{~nm}$ 的通道 ${ }^{[20]}$ (如图 2 所示). 他们将聚(3,4-亚乙二氧基噻吩)-聚(苯乙烯磺 酸)(PEDOT/PSS) 墨滴喷射至图案化基材表面, 借助其 浸润性差异，同时通过控制液滴的粘度以及体积，诱导 墨滴在疏水区去浸润, 得到与疏水图案相当宽度的空白 区.

Jung ${ }^{[21]}$ 等利用液滴蒸发过程中的“咖啡环”效应，使 溶质只在液滴的边缘沉积, 得到了宽度为 $1.14 \mu \mathrm{m}$ 的双 连续线条以及线宽为 $250 \mathrm{~nm}$ 的三角形图案. 如图 $3 \mathrm{a}$ 所 
(a) (i)

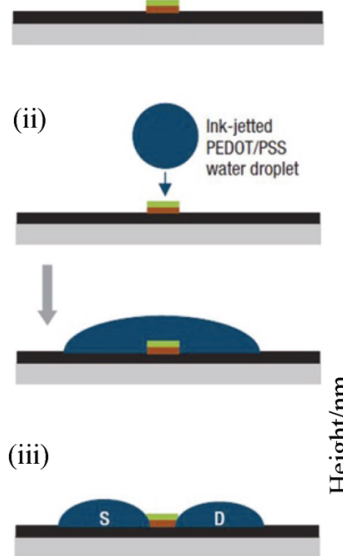

(b)

(c) 0
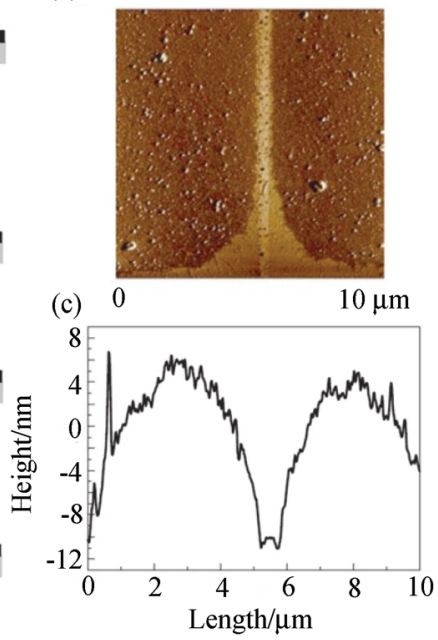

图 2 喷墨液滴在图案化表面去浸润. (a)去浸润过程. (b) PEDOT/PSS 墨滴在图案化基材表面去浸润后的原子力显微镜相图及其(c)断面图 Figure 2 Dewetting of inkjet droplets on patterned surfaces. (a) The dewetting process. (b) AFM phase image and (c) AFM cross-sectional image of dewetted PEDOT/PSS droplets (a)

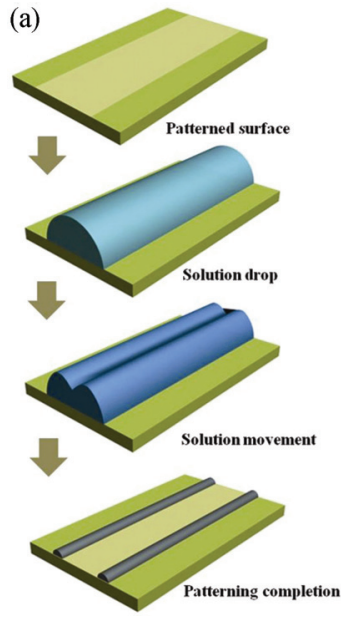

(b)

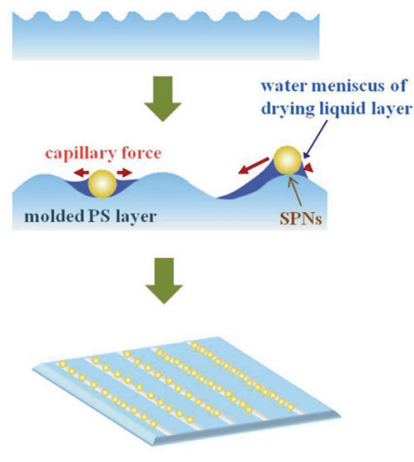

图 3 (a)液滴边缘去浸润刻蚀示意图. (b)聚合物半导体纳米微球在聚 合物模板中组装形成功能纳米结构的过程示意图. 将含有聚合物半导 体纳米微球的墨水打印至具有凹槽结构的基材表面. 由于毛细力的作 用, 液滴蒸发过程中微球在基材的凹槽中组装

Figure 3 (a) Schematic of dewetting edge lithography. (b) Schematic of the fabrication process for functional nanostructures from assembled semiconducting polymer nanospheres (SPNs) in a polymeric template. The dispersion containing the SPNs is inkjet printed onto this surface. Due to capillary forces acting on the spheres, they finally assemble in the grooves of the template while the drop dried

示, 在亲/疏水图案化的基材表面, 液滴自发在亲水区铺 展. 液滴蒸发过程中, 溶质随着液滴内部的毛细流动移 动至液滴边缘并沉积, 而液滴中心没有残留溶质. 由此 在液滴边缘形成了纳米尺寸的连续线条. 该方法为利用 液滴去浸润现象提高喷墨打印图案分辨率提供了新思 路.

不仅基材表面的浸润性差异可以诱导墨滴的浸润/ 去浸润行为, 其不同物理结构同样可以诱导墨滴的浸润
行为, 控制液滴的铺展. Schubert 等 ${ }^{[22]}$ 利用基材的物理 限域作用, 制备了宽度为 $15 \mu \mathrm{m}$ 的连续银导线. 他们采 用热压的方法在聚合物基材表面构建了数微米宽的凹 槽. 当含有银纳米粒子的墨滴喷射至基材表面时，由于 毛细力的作用液滴自动向凹槽处铺展, 并最终在凹槽底 部沉积. List 等 ${ }^{[23]}$ 采用类似的方法在聚合物基材表面构 建了模板(图 3b). 当墨滴在模板上干燥时, 由于液滴弯 液面的毛细力作用，墨滴中的聚合物半导体纳米微球在 模板的凹槽部分组装, 得到了连续的窄线条.

\section{3 改进喷墨设备}

墨滴的体积大小决定了液滴在基材上的沉积面积. 通常，从喷孔喷出的墨滴体积越小，其在基材上的铺展 面积越小, 喷墨打印图案的分辨率越高. 墨滴的体积主 要由喷孔大小决定. 减小喷孔的孔径可以直接减小墨滴 的体积, 有效提高打印图案的分辨率. 但是, 当喷孔的 孔径减小到一定尺寸后，现有的基于压电或热泡技术的 喷墨打印设备无法克服细小喷孔处的巨大的毛细作用 力以及粘度效应, 不能将液滴从喷孔处喷射出. 因此, 为了突破现有普通喷墨打印机的限制，进一步提高打印 分辨率, 科研人员对喷墨设备进行了设计和改进.

为了得到纳米尺寸的微小墨滴, Rogers 课题组 ${ }^{[24]}$ 发 展了电流体力学喷墨打印技术(图 4). 该技术可将墨水 从 $300 \mathrm{~nm}$ 的毛细管中喷出, 得到直径为 $(240 \pm 50) \mathrm{nm}$ 的墨滴, 并打印出分辨率为数百纳米的图案和功能器 件. 具体过程是, 在喷头和导电基底间施加电压, 产生 的电场引起墨水中的离子聚集于喷头的弯液面附近. 这 些离子间的库仑排斥力导致液体表面产生切向应力, 从 而使弯液面变形为圆雉状, 称为泰勒雉. 在合适的电场 下, 泰勒雉顶点处的静电压力克服毛细张力, 纳米尺寸 的液滴会从顶点喷出. 随后，该课题组用该喷墨打印设 备制备了具有纳米尺寸分辨率的带正电荷及负电荷的 复杂图案 ${ }^{[25]}$. 该方法是目前分辨率最高的喷墨打印方 法, 并且可以实现大面积精密图案的制备, 对于发展新 型的喷墨打印设备具有重要的借鉴意义.

Espinosa 等 ${ }^{[26]}$ 利用尺寸为 $400 \mathrm{~nm}$ 的原子力显微镜 输送探针针尖作为喷头, 制备了最小尺寸为 $93 \mathrm{~nm}$ 的点 阵图案. 当探针与基材接触时, 墨水在毛细力作用下从 纳米尺寸的原子力显微镜探针喷出. 墨滴尺寸可通过控 制喷头与基材的接触时间来调控. 该方法为原子力显微 镜探针在高分辨率图案制备方面的应用提供了新思路. Lovsky 等 ${ }^{[27]}$ 将电泳与原子力显微镜探针结合打印牛血 清蛋白, 制备了宽度为 $160 \sim 170 \mathrm{~nm}$ 的连续线. 施加的 脉冲电压可精确控制蛋白质分子的运动进而控制墨水 的输出, 而原子力显微镜探针可精确控制打印位置.

除了减小喷孔直径，还可以通过改变喷射原理提 高图案分辨率. Ferraro 课题组 ${ }^{[28]}$ 开发了一种基于热电效 应的喷射技术，从直径为 $6 \mu \mathrm{m}$ 的母液滴中喷射出直径 
(a)

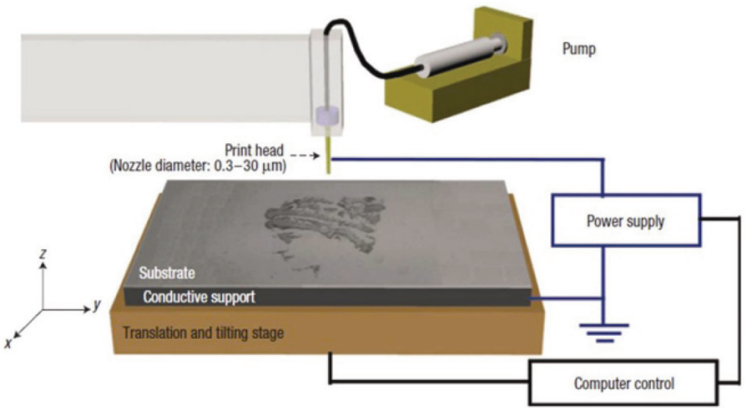

(b)

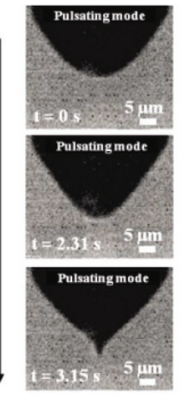

(c)

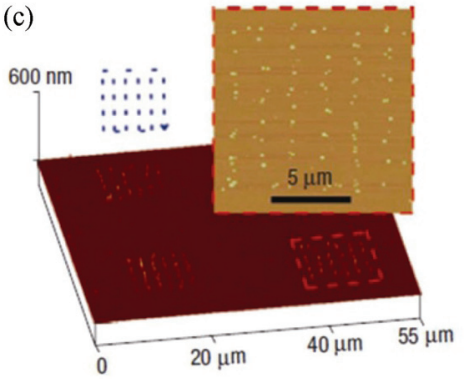

图 4 利用电流体力学实现高分辨率喷墨打印. (a)打印设备装置图. (b)脉冲弯液面随时间变化的微速摄影照片. (c) 点阵列图案的三维原 子力显微镜照片, 点直径为 $(240 \pm 50) \mathrm{nm}$, 所用喷孔内径为 $300 \mathrm{~nm}$

Figure 4 High-resolution electrohydrodynamic jet printing. (a) Printer set-up. (b) Time-lapse optical microscopy images of the pulsating liquid meniscus. (c) Three-dimensional AFM image of aligned arrays of dots with diameters of $(240 \pm 50) \mathrm{nm}$, formed using a 300-nm-internal-diameter nozzle

为 $300 \mathrm{~nm}$ 的子液滴. 具体原理如图 5a 所示, 由于铌酸 锂材料特殊的热电效应，当施加红外激光或点热源时， 液滴的上方产生电场, 由此诱导液滴顶部形成圆锥状尖 端. 当尖端液膜破裂, 液滴便喷射出体积只有母液滴 $0.5 \%$ 的子液滴. 该方法可以利用母液滴的体积调控子 液滴的尺寸，同时通过移动红外激光或点热源的位置实 现图案制备. Gañán-Calvo 等 ${ }^{[29]}$ 采用双重流动聚焦原理 得到了最小尺寸为 $600 \mathrm{~nm}$ 的液滴. 其装置如图 $5 \mathrm{~b}$ 所 示, 从内径为 $150 \mu \mathrm{m}$ 毛细管流出的录由另一种高速运 动的硅油驱动, 同时再由最外层的高速运动的气流驱 动, 经小孔聚焦后形成稳定的圆雉, 在圆雉的顶端产生 一股微射流穿过小孔，射流因不稳定性破碎成单分散性 的直径为 $600 \mathrm{~nm}$ 的录微滴.

通过优化墨水的化学组成、调控基材表面的化学组 成或物理结构以及改进喷射设备可以有效地提高喷墨 打印图案的分辨率. 这些方法对于发展高分辨率喷墨打 印技术具有重要意义.

\section{3 抑制 “咖啡环” 效应}

喷墨打印中功能溶质的均匀沉积对高精度图案的 形成及所制备器件的性能与应用都非常重要. 抑制喷墨 墨滴的 “咖啡环” 效应实现均质打印是提高图案精度的 关键问题之一. 这里对抑制 “咖啡环” 效应的研究工作 进行总结, 涉及的内容包括 “咖啡环” 形成机理以及消

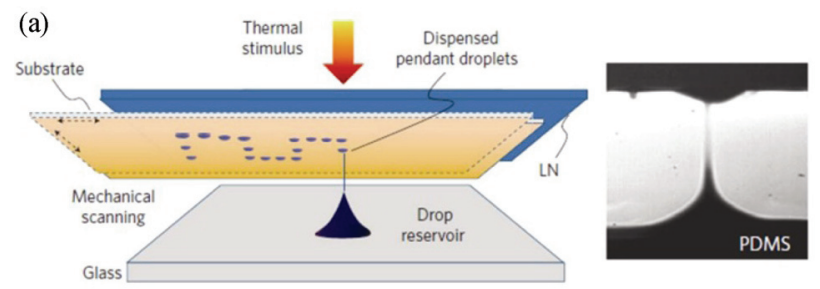

(b)

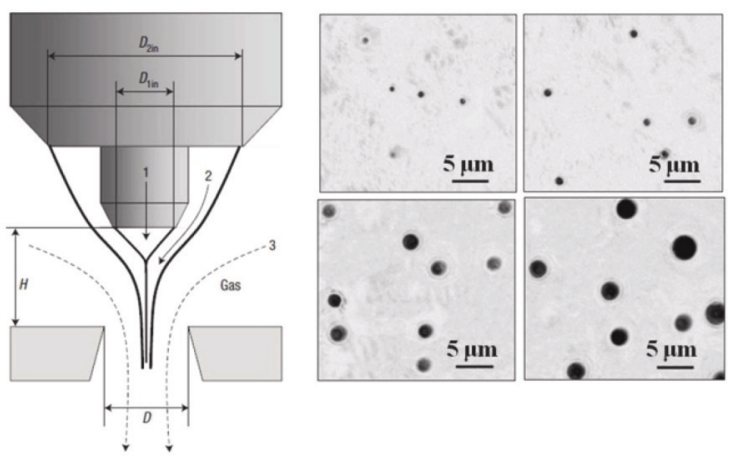

图 5 (a)通过喷射纳升体积的墨滴制备图案的原理示意图. 墨滴喷射 至铌酸锂与玻璃片之间的基材上. (b)双流动聚焦装置示意图

Figure 5 (a) Schematic of dispensing nanolitre droplets for liquid patterning. Liquid is dispensed onto a translating substrate inserted between the lithium niobate and the glass plate. (b) The double fluidic focusing arrangement

除 “咖啡环” 效应的方法.

“咖啡环” 是喷墨打印墨滴的一种常见沉积形式. 当墨滴在基材表面扩散蒸发时，其中的溶质通常会沿着 液滴的边缘沉积，形成不均匀的环状形貌. 这会大大降 低喷墨打印图案的精度，限制了喷墨打印技术在高性能 器件制备方面的应用. 在过去的十几年中, 科研人员对 形成 “咖啡环” 的机理以及液滴内部的毛细流动进行了 深入的研究. Deegan 等 ${ }^{[30 ~ 32]}$ 最早认为, “咖啡环” 的形 成是由于蒸发过程中，液滴的边缘固定在固体表面，而 边缘处液滴的挥发速率大于液滴中心处的挥发速率, 由 此在液滴内部产生了由中心流向边缘的毛细流动, 将悬 浮的粒子携带至液滴边缘. 当蒸发结束时, 悬浮的粒子 集中沉积在液滴边缘, 形成 “咖啡环” (图 6a). Hu 和 Larson $^{[33]}$ 进一步指出, 除了外向的毛细流动, 蒸发过程 中液滴内部还存在一个沿着液滴表面、由表面张力梯度 差引起的从液滴边缘向中心的流动, 称为马拉哥尼流 动, 该流动有利于促使悬浮粒子向液滴中心移动(图 6b).

液滴的尺寸对于 “咖啡环” 的形成会产生影响, “咖 啡环” 效应存在一个尺寸极限 ${ }^{[34]}$. 随着液滴尺寸的减小, 液滴蒸发的速度会大大增加, 而液滴内粒子的运动速度 却变化不大. 当液滴的尺寸小到一定程度, 液滴蒸发的 速度将远远大于粒子的运动速度. 在液滴蒸发完之前, 粒子来不及运动至液滴边缘便沉积. 研究结果显示, 对 于直径为 $100 \mathrm{~nm}$ 的纳米颗粒, 形成 “咖啡环” 的最小 液滴尺寸为 $10 \mu \mathrm{m}$.

基于对 “咖啡环” 形成机理的认识, 研究人员通常 

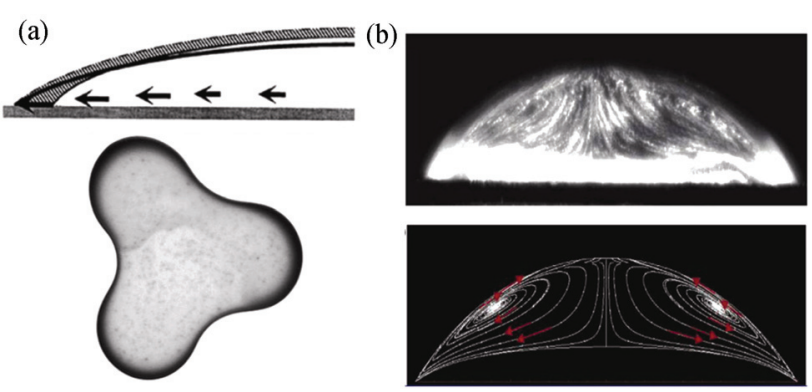

图 6 (a) “咖啡环” 沉积形貌. 上: 蒸发过程中外向毛细流动使三相 接触线固定的机理. 下: 溶剂蒸发完毕后形成的 “咖啡环”. (b)辛烷液 滴干燥过程中其内部流场的照片(上)及其模拟结果(下)

Figure 6 (a) "Coffee ring" deposits. Top: the mechanism of outward flow during evaporation to keep the contact line fixed. Bottom: a "coffee ring" formed after evaporation. (b) Flow field in a drying octane droplet, (top) imaged experimentally and (bottom) predicted

采用两类方法来控制 “咖啡环” 效应. 一是通过减弱液 滴内部的外向毛细流动或增大马拉哥尼流动来控制液 滴内部的毛细流动; 二是控制蒸发过程中液滴三相接触 线的移动.

\section{1 控制液滴内部的毛细流动}

墨滴在基材表面的蒸发过程非常复杂, 始终处于非 平衡态. 墨滴内部的毛细流动是形成 “咖啡环” 的关键, 因而近年来大量研究工作致力于控制液滴内部的毛细 流动, 以消除或改善 “咖啡环” 效应.

由于液滴内部存在的外向毛细流动将粒子携带至 液滴边缘是形成 “咖啡环” 的原因, 因此减弱外向毛细 流动可以有效抑制 “咖啡环” 效应. Yodh 等 ${ }^{[35]}$ 利用椭球 形粒子间的毛细作用力消除了液滴干燥过程中 “咖啡 环” 的形成. 当椭球形粒子被外向毛细流动带至空气一 水界面后, 粗球形粒子使液面发生显著变形, 从而产生 较强的粒子间毛细作用力. 这种强且远程的粒子间吸引 力使椭球形粒子在空气一水界面形成松散聚集结构, 进 而导致液滴表层的粘度迅速增大, 阻止了悬浮的粒子移 向液滴边缘, 确保形成均匀的沉积(图 7a). 有意思的是, 在合适的条件下, 混有少量椭球形粒子的球形粒子悬浮 液也能形成均匀的沉积形貌. 除了椭球形粒子以外, 棒 状、丝状 ${ }^{[35]}$ 以及片状 ${ }^{[36]}$ 的粒子都可以抑制 “咖啡环” 的 形成. 随后, 该课题组又利用离子型表面活性剂十二烷 基硫酸钠调控液滴内部的毛细流动, 得到相对均匀的粒 子沉积膜 ${ }^{[37]}$. 当液滴中加入少量的离子型表面活性剂 时, 外向的毛细流动将表面活性剂带至液滴边缘并在 液一气界面形成一层单分子层膜. 液滴边缘的表面张力 降低, 引发了由液滴边缘流向液滴中心的马拉哥尼回 流. 该回流与外向毛细流动共同作用, 在液滴边缘形成 了马拉哥尼漩浴层, 阻止了液滴中的粒子在边缘沉积.

蒸发液滴中的粒子在蒸发流的作用下逐渐迁移至 液滴表面, 利用粒子在蒸发液滴的表面形成单粒子层膜 可以有效抑制粒子向液滴边缘移动. Jaeger 等 ${ }^{[38]}$ 在金纳 米粒子悬浮液中加入十二硫醇, 通过控制液滴蒸发动力 (a)

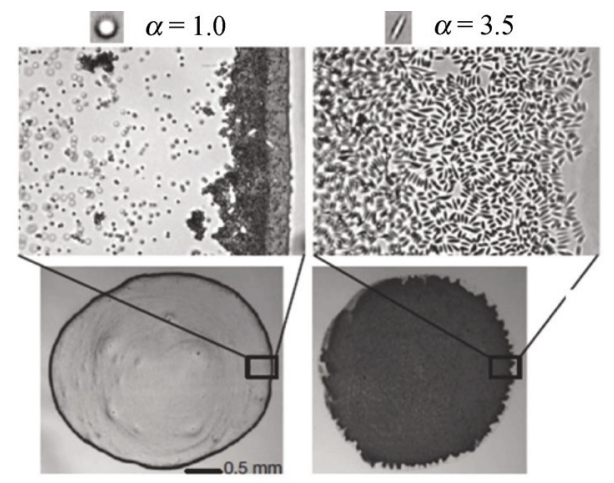

(b)

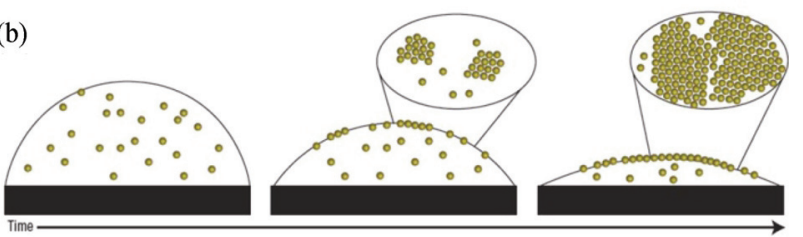

图 7 (a)含有球形粒子(左)和椭球形粒子(右)的液滴蒸发至一半时, 距其三相接触线 $40 \mu \mathrm{m}$ 范围内的光学显微镜照片. (b)液滴蒸发早期粒 子组装过程示意图

Figure 7 (a) Microscope images of a region within $40 \mu \mathrm{m}$ of the drop contact line, taken at time $t / t_{\text {final }}=0.5$, for suspensions of spheres (left) and ellipsoids (right). (b) Schematic diagram of the self-assembly process during the early stages of drying

学过程以及粒子与液面的毛细作用力, 使金纳米粒子在 液面形成单粒子层. 形成单粒子层的金纳米粒子被固定 在液滴的表面，无法重新运动至液滴内部，同时也难以 向液滴边缘移动, 从而有效地抑制了 “咖啡环” 的形成 (图 7b).

不仅粒子在液面形成的结构可以改变粒子在液滴 中的毛细流动方向, 粒子与液面或者粒子与基底之间的 作用力也可以显著改变粒子在液滴中的毛细流动方向. Jung ${ }^{[39]}$ 等发现粒子与液面间的毛细作用力可以改变粒 子在液滴中的运动方向. 当粒子尺寸较小时, 粒子在液 滴的三相接触线处沉积, 形成 “咖啡环” ; 而当粒子尺 寸较大时, 粒子与液面的作用力较大, 液滴蒸发过程中 液面移动改变粒子的运动方向, 迫使粒子向液滴中心移 动(图 8a). 此外, Attinger 等 ${ }^{[40]}$ 通过调节液体的 $\mathrm{pH}$ 值, 调 控粒子与基底之间的静电作用力以及范德华力, 控制粒 子在液滴中的运动方向, 进而控制粒子的最终沉积形貌 (图 8b). 当 $\mathrm{pH}$ 值小于 5.8 时, 粒子与基底间的吸引力占 主导, 粒子运动至液滴边缘之前已沉积至基底, 粒子沉 积形貌相对均匀; 当 $\mathrm{pH}$ 值介于 5.8 至 8.9 之间时，马拉 哥尼回流占主导, 粒子被携带至液滴中心沉积; 当 $\mathrm{pH}$ 值大于 8.9 时, 粒子与基底间的排斥力占主导, 粒子容 易运动至液滴边缘形成 “咖啡环” .

其他方法也可以控制干燥过程中液滴内部的毛细 流动. 例如, Subramanian 等 ${ }^{[41]}$ 采用降低温度的方法减弱 外向毛细流动. 他们发现降低基底的温度, 液滴的蒸发 速率下降. 但边缘蒸发速率下降程度比中心的大, 减弱 了液滴边缘与中心的蒸发速率差, 粒子向边缘移动的趋 
(a)

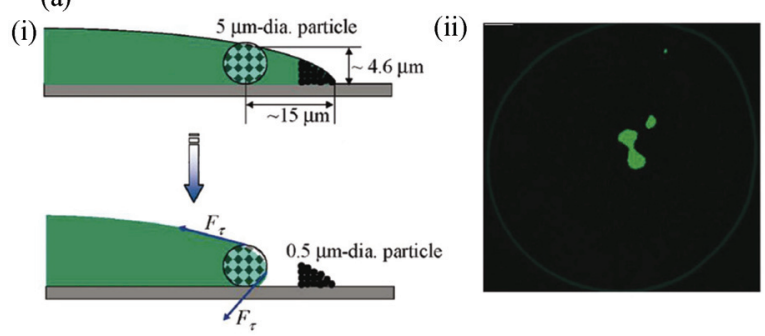

(b) DLVO attractive force Marangoni recirculation Radial flow

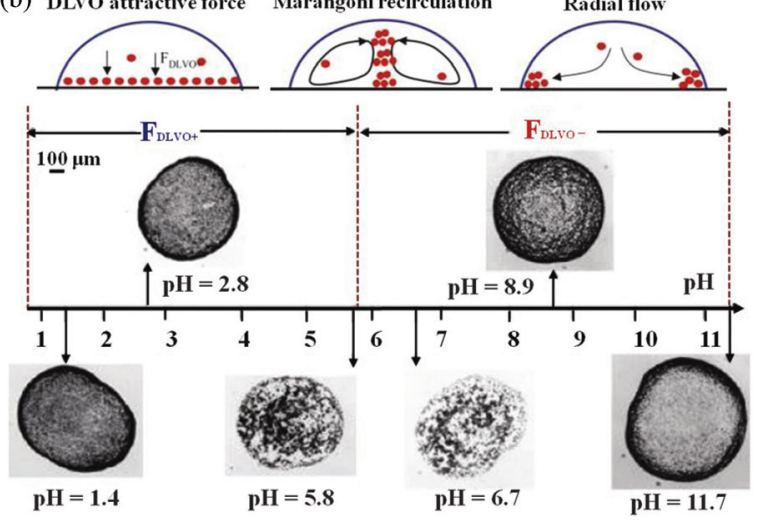

图 8 (a) (i)液滴中粒子所处位置示意图. 去浸润后的三相线移向粒径 为 $5 \mu \mathrm{m}$ 粒子的表面, 其中 $F_{\tau}$ 表示作用在单个粒子上的表面张力. (ii) 含有 $0.005 \mathrm{vol} \%$ 粒径为 $0.5 \mu \mathrm{m}$ 的粒子和 $0.005 \mathrm{vol} \%$ 粒径为 $5 \mu \mathrm{m}$ 的粒 子的液滴中, 两种粒子几乎完全分离. 粒径为 $0.5 \mu \mathrm{m}$ 的粒子形成环状 沉积, 而粒径为 $5 \mu \mathrm{m}$ 的粒子在中心沉积. (b) 粒子与基底毛细作用力 控制粒子运动. 上: 三种毛细流动机理相互竞争决定沉积形貌. 下: 不同 $\mathrm{pH}$ 值条件下形成的沉积形貌

Figure 8 (a) (i) Schematic diagram of particle positions in the droplet. Depinned contact line moves to the surface of $5 \mu \mathrm{m}$ diameter particles, where surface tensile forces $\left(F_{\tau}\right)$ act on individual particles. (ii) For the mixture consisting of $0.005 \mathrm{vol} \%$ of $0.5 \mu \mathrm{m}$ diameter and $0.005 \mathrm{vol} \%$ of 5 $\mu \mathrm{m}$ diameter particles, one can see the quasi-perfect separation of $0.5 \mu \mathrm{m}$ diameter particles and $5 \mu \mathrm{m}$ diameter particles. The ring corresponds to $0.5 \mu \mathrm{m}$ diameter particles, while $5 \mu \mathrm{m}$ diameter particles accumulate around the center. (b) The capillary force between the particle and the substrate tuning particle deposition morphology. Top: three convective mechanisms compete to form the deposit. Bottom: deposit patterns obtained for different values of $\mathrm{pH}$

势减弱. 当温度为 $17^{\circ} \mathrm{C}$ 时, “咖啡环” 现象消失. Harris 等 ${ }^{[2]}$ 通过控制液滴的蒸发流调控液滴内部的毛细流动。 他们将含有粒子的液滴置于带有微米尺寸的多孔模板 下蒸发, 液滴表面的蒸发速率随多孔模板出现周期性变 化. 在蒸发流的作用下，液滴中的粒子向微孔处移动而 不是向液滴边缘移动. 因此, 在适当的粒子浓度下可形 成均匀的沉积形貌. Culha 等 $^{[43]}$ 在液滴的中心插入一根 毛细管，通过调控毛细管表面的浸润性改变粒子在毛细 管表面的沉积状态, 从而调控液滴内部的毛细流动, 控 制粒子的沉积形貌.

$\mathrm{Hu}$ 和 $\mathrm{Larson}^{[33]}$ 提出, 蒸发过程中的液滴表面存在 马拉哥尼流动. 这是由于干燥过程中液滴中心与液滴边 缘的温度差引起表面张力梯度差, 引发了液体从低表面 张力处流向高表面张力处. 通常情况下, 马拉哥尼流动 是沿着液滴表面从液滴边缘向中心流动的, 与蒸发引起
的液滴内部外向毛细流动方向相反，因此增大马拉哥尼 流动可以有效控制 “咖啡环” 效应. 改变溶剂组成是常 用的方法 ${ }^{[44 ~ 48]}$, 在液滴中加入高沸点、低表面张力的第 二组分溶剂, 可以增大这一流动. 如图 9 所示, Moon 等 ${ }^{[45]}$ 在溶液中添加高沸点、低表面张力的第二组分溶剂乙二 醇. 由于水在液滴边缘的蒸发速率大于液滴中心, 而乙 二醇挥发速率较慢, 因此随着蒸发过程的进行, 液滴边 缘乙二醇浓度会逐渐高于液滴中心乙二醇浓度. 乙二醇 的浓度差进一步降低了液滴边缘的表面张力, 使液滴边 缘与中心产生了附加的表面张力差, 增大了内部的马拉 哥尼流动. 实验结果显示, 随着乙二醇含量的增加, 粒 子的沉积形貌变得更均匀. 当乙二醇的质量分数为 $32 \%$ 时, “咖啡环” 现象消失. 值得注意的是, 并不是任何情 况下液滴表面的马拉哥尼流动都是从液滴边缘流向中 心的. Stone 等 ${ }^{[49]}$ 发现基底的导热性会显著影响液滴内 部的马拉哥尼流动, 从而影响粒子的沉积形貌. 当基底 的导热系数与溶剂的导热系数比值大于 2 时, 马拉哥尼 流动由内向外，而当其比值小于 1.45 时，马拉哥尼流动 由外向内. (a)

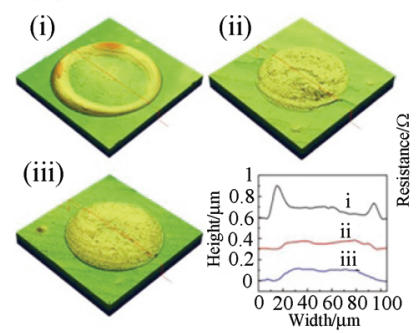

(b)

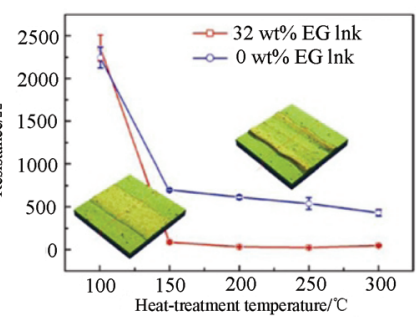

图 9 溶剂组成对液滴沉积形貌的影响. (a)不同乙二醇含量的墨滴沉 积后的三维共聚焦显微镜照片. 其中乙二醇质量分数分别为 (i) $0 \%$, (ii) $16 \%$, (iii) $32 \%$. (b)打印导电线的电阻随处理温度的变化关系, 所 用墨水中乙二醇质量分数分别为 $0 \%$ 和 $32 \%$. 其中插图为所打印导电 线路的三维共聚焦显微镜照片

Figure 9 Improvement of film morphology by controlling solvent compositions. (a) Confocal laser scattering microscopic three-dimensional images for ink-jet printed single dots obtained from inks with varying solvent compositions: (i) 0, (ii) 16, (iii) $32 \mathrm{wt} \%$ ethylene glycol. (b) Resistance of the ink-jet printed lines as a function of heat-treatment temperature for 0 and $32 \mathrm{wt} \%$ ethylene glycol inks. Insets show three-dimensional confocal microscopic images of the ink-jet printed lines

\section{2 控制液滴三相接触线的移动}

喷墨墨滴在蒸发过程中三相接触线固定在基底表 面是形成 “咖啡环” 的另一必要条件. 基底一般不是理 想的光滑表面, 通常存在许多物理或化学缺陷. 当墨滴 在基底表面挥发时，其三相接触线往往会因为这些缺陷 的存在而固定在基底表面. 虽然墨滴蒸发初期三相接触 线对基底的粘附力较小，但是随着蒸发过程的进行，墨 滴中的粒子会逐渐移动至三相接触线处并沉积, 进一步 地将三相接触线固定在基底上. 施加一定的外界作用力 使墨滴蒸发过程中三相接触线随着液滴体积减小而不 
断移动, 可使粒子难以在墨滴边缘沉积, 达到抑制 “咖 啡环” 的目的.

最近, Augustine 等 ${ }^{[50]}$ 采用电润湿的方法抑制 “咖啡 环” 的形成(图 10). 他们在蒸发的液滴上施加交流电压, 一方面使液滴蒸发过程中三相接触线在基底上不固定, 粒子随着三相接触线的回缩向液滴中心移动; 另一方面 交流电压使液滴内部产生了由外向内的毛细流动, 使液 滴内部的粒子向中心移动. 两方面共同作用使 “咖啡环” 效应得到了有效的控制，最终形成均匀的沉积膜.

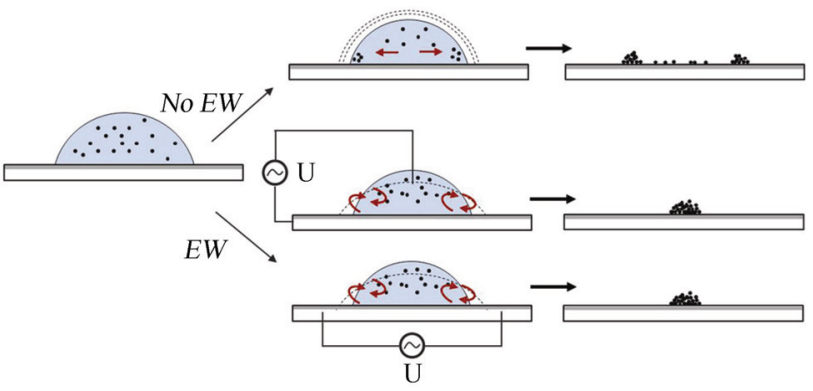

图 10 加电压和不加电压时液滴蒸发过程示意图. 不加电压时液滴 蒸发过程中三相接触线固定(上); 加电压时液滴蒸发过程中三相接触 线移动(中和下)

Figure 10 Schematic of the drop evaporation process with and without electrowetting. Top row represent evaporation without electrowetting and with contact line pinning; middle and bottom panels show the process with electrowetting and mobile contact lines

\section{4 总结与展望}

作为最具应用前景的图案化方法之一，喷墨打印技 术具有大面积直写、环境友好、成本低廉、功能多样化 等优势. 通过精确调控墨滴在基材表面的浸润铺展行为 以及不断改进喷墨打印喷射技术，已实现了喷墨打印图 案分辨率从微米到纳米尺度的跨跃, 使得喷墨打印技术 在微半导体、光电器件、生物器件等功能器件制备方面 的应用更为令人瞩目. 但到目前为止, 喷墨打印技术还 未大规模用于功能器件的制备, 其原因在于喷墨打印材 料以及高精度喷墨打印设备还有诸多不完善之处. 例 如, 目前实现高精度图案的打印需使用特殊功能材料或 对基材的化学物理结构进行预处理, 一些特殊的高精度 喷墨打印设备的成本较高, 使用不便, 这在很大程度上 限制了喷墨打印技术在高精度图案及其功能器件制备 领域的应用.

针对以上存在的诸多问题, 开发新型喷墨打印功能 材料、优化基材物理化学结构的构筑以及发展更简便的 高精度打印设备显得尤为重要. 随着科学技术的发展, 特别是纳米材料和纳米技术的研究不断深入，喷墨打印 技术也展现出更为广阔的应用前景. 相信随着相关物 理、化学基础研究的不断深入和新技术、新材料的不断 发展, 喷墨打印技术在高精度图案及其功能器件的制备 领域将得到日益广泛的应用.
作者简介

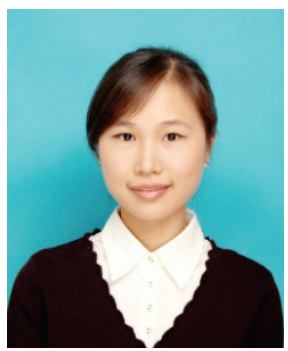

户旻詈, 中国科学院化学研究所 2011 级在读博士生. 2009 年获四川大学硕士学位. 研究方向为液滴可控去浸润.

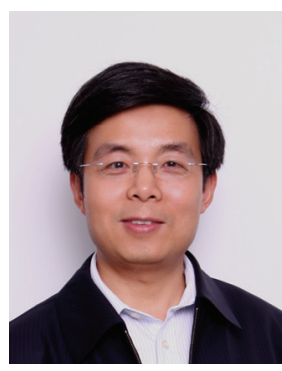

宋延林, 中国科学院化学研究所研究员. 1996 年获北京大 学理学博士学位; 1996 1998 清华大学化学系博士后. 主要研 究方向为高密度信息存储薄膜、聚合物光子晶体及绿色打印 印刷材料与技术研究. 已发表论文 160 余篇, 主持和参加编写 英文专著 5 部，中文专著 1 部，获 2008 年和 2005 年国家自然 科学二等奖等.

\section{References}

[1] Hwang, J. K.; Cho, S.; Dang, J. M.; Kwak, E. B.; Song, K.; Moon, J.; Sung, M. M. Nat. Nanotechnol. 2010, 5, 742.

[2] Tan, C. P.; Cipriany, B. R.; Lin, D. M.; Craighead, H. G. Nano Lett. 2010, 10, 719.

[3] Minari, T.; Liu, C.; Kano, M.; Tsukagoshi, K. Adv. Mater. 2012, 24, 299.

[4] Ito, T.; Okazaki, S. Nature 2000, 406, 1027.

[5] Geissler, M.; Xia, Y. N. Adv. Mater. 2004, 16, 1249.

[6] Huo, F. W.; Zheng, Z. J.; Zheng, G. F.; Giam, L. R.; Zhang, H.; Mirkin, C. A. Science 2008, 321, 1658.

[7] Xu, H.; Ling, X. Y.; van Bennekom, J.; Duan, X.; Ludden, M. J. W.; Reinhoudt, D. N.; Wessling, M.; Lammertink, R. G. H.; Huskens, J. J. Am. Chem. Soc. 2009, 131, 797 .

[8] Kraus, T.; Malaquin, L.; Schmid, H.; Riess, W.; Spencer, N. D.; Wolf, H. Nat. Nanotechnol. 2007, 2, 570.

[9] Tekin, E.; Smith, P. J.; Schubert, U. S. Soft Matter 2008, 4, 703.

[10] Zhang, L.; Liu, H. T.; Zhao, Y.; Sun, X. N.; Wen, Y. G.; Guo, Y. L.; Gao, X. K.; Di, C. A.; Yu, G.; Liu, Y. Q. Adv. Mater. 2012, 24, 436.

[11] van den Berg, A. M. J.; de Laat, A. W. M.; Smith, P. J.; Perelaer, J.; Schubert, U. S. J. Mater. Chem. 2007, 17, 677.

[12] van Osch, T. H. J.; Perelaer, J.; de Laat, A. W. M.; Schubert, U. S. Adv. Mater. 2008, 20, 343.

[13] Sele, C. W.; von Werne, T.; Friend, R. H.; Sirringhaus, H. Adv. Mater. 2005, 17, 997.

[14] Noh, Y. Y.; Zhao, N.; Caironi, M.; Sirringhaus, H. Nat. Nanotechnol. 2007, 2, 784 .

[15] Cui, L. Y.; Li, Y. F.; Wang, J. X.; Tian, E. T.; Zhang, Y. Z.; Song, Y. L.; Jiang, L. J. Mater. Chem. 2009, 19, 5499.

[16] Li, Z. R.; Wang, J. X.; Zhang, Y. Z.; Wang, J. J.; Jiang, L.; Song, Y. L. Appl. Phys. Lett. 2010, 97, 233107.

[17] Bai, C. L. Chin. Sci. Bull. 2009, 54, 1941. (白春礼, 科学通报, 2009, 54, 1941.)

[18] Zhou, H. H.; Song, Y. L. Adv. Mater. Res. 2011, 174, 447. 
[19] Sirringhaus, H.; Kawase, T.; Friend, R. H.; Shimoda, T.; Inbasekaran, M.; Wu, W.; Woo, E. P. Science 2000, 290, 2123.

[20] Wang, J. Z.; Zheng, Z. H.; Li, H. W.; Huck, W. T. S.; Sirringhaus, H. Nat. Mater. 2004, 3, 171.

[21] Lee, K. H.; Kim S. M.; Jeong, H.; Jung, G. Y. Soft Matter 2012, 8, 465.

[22] Hendriks, C. E.; Smith, P. J.; Perelaer, J.; Van den Berg, A. M. J.; Schubert, U. S. Adv. Funct. Mater. 2008, 18, 1031.

[23] Fisslthaler, E.; Blumel, A.; Landfester, K.; Scherf, U.; List, E. J. W. Soft Matter 2008, 4, 2448.

[24] Park, J. U.; Hardy, M.; Kang, S. J.; Barton, K.; Adair, K.; Mukhopadhyay, D. K.; Lee, C. Y.; Strano, M. S.; Alleyne, A. G.; Georgiadis, J. G.; Ferreira, P. M.; Rogers, J. A. Nat. Mater. 2007, 6, 782.

[25] Park, J. U.; Lee, S.; Unarunotai, S.; Sun, Y. G.; Dunham, S.; Song, T.; Ferreira, P. M.; Alleyene, A. G.; Paik, U.; Rogers, J. A. Nano Lett. 2010, 10, 584

[26] Loh, O.; Lam, R.; Chen, M.; Moldovan, N.; Huang, H. J.; Ho, D.; Espinosa, H. D. Small 2009, 5, 1667.

[27] Lovsky, Y.; Lewis, A.; Sukenik, C.; Grushka, E. Anal. Bioanal. Chem. 2010, 396, 133.

[28] Ferraro, P.; Coppola, S.; Grilli, S.; Paturzo, M.; Vespini, V. Nat. Nanotechnol. 2010, 5, 429.

[29] Ganan-Calvo, A. M.; Gonzalez-Prieto, R.; Riesco-Chueca, P.; Herrada, M. A.; Flores-Mosquera, M. Nat. Phys. 2007, 3, 737.

[30] Deegan, R. D.; Bakajin, O.; Dupont, T. F.; Huber, G.; Nagel, S. R.; Witten, T. A. Nature 1997, 389, 827.

[31] Deegan, R. D. Phys. Rev. E 2000, 61, 475.

[32] Deegan, R. D.; Bakajin, O.; Dupont, T. F.; Huber, G.; Nagel, S. R.; Witten, T. A. Phys. Rev. E 2000, 62, 756.
[33] Hu, H.; Larson, R. G. J. Phys. Chem. B 2006, 110, 7090.

[34] Shen, X.; Ho, C. M.; Wong, T. S. J. Phys. Chem. B 2010, 114, 5269.

[35] Yunker, P. J.; Still, T.; Lohr, M. A.; Yodh, A. G. Nature 2011, 476, 308.

[36] Hodges, C. S.; Ding, Y.; Biggs, S. J. Colloid Interface Sci. 2010, $352,99$.

[37] Still, T.; Yunker, P. J.; Yodh, A. G. Langmuir 2012, 28, 4984.

[38] Bigioni, T. P.; Lin, X. M.; Nguyen, T. T.; Corwin, E. I.; Witten, T. A.; Jaeger, H. M. Nat. Mater. 2006, 5, 265.

[39] Jung, J. Y.; Kim, Y. M.; Yoo, J. Y. Anal. Chem. 2009, 81, 8256.

[40] Bhardwaj, R.; Fang, X. H.; Somasundaran, P.; Attinger, D. Langmuir 2010, 26, 7833 .

[41] Soltman, D.; Subramanian, V. Langmuir 2008, 24, 2224.

[42] Harris, D. J.; Hu, H.; Conrad, J. C.; Lewis, J. A. Phys. Rev. Lett. 2007, 98, 148301 .

[43] Keseroglu, K.; Culha, M. J. Colloid Interface Sci. 2011, 360, 8.

[44] Park, J.; Moon, J. Langmuir 2006, 22, 3506.

[45] Kim, D.; Jeong, S.; Park, B. K.; Moon, J. Appl. Phys. Lett. 2006, 89, 264101.

[46] Denneulin, A.; Bras, J.; Carcone, F.; Neuman, C.; Blayo, A. Carbon 2011, 49, 2603

[47] Tekin, E.; de Gans, B. J.; Schubert, U. S. J. Mater. Chem. 2004, 14, 2627.

[48] de Gans, B. J.; Schubert, U. S. Langmuir 2004, 20, 7789

[49] Ristenpart, W. D.; Kim, P. G.; Domingues, C.; Wan, J.; Stone, H. A. Phys. Rev. Lett. 2007, 99, 234502.

[50] Eral, H. B.; Augustine, D. M.; Duits, M. H. G.; Mugele, F. Soft Matter 2011, 7, 4954. 